\title{
Comparison of Vibrating Mesh, Jet, and Breath-Enhanced Nebulizers During Mechanical Ventilation
}

\author{
Sunya Ashraf, Michael McPeck, Ann D Cuccia, and Gerald C Smaldone
}

\begin{abstract}
BACKGROUND: This study compared 3 nebulizer technologies for inter- and intradevice reproducibility, humidification, and fill volume sensitivity during mechanical ventilation: a breathenhanced jet nebulizer, a vibrating mesh nebulizer, and a jet nebulizer. The breath-enhanced jet nebulizer featured a new design located on the wet side of the humidifier to reduce aerosol loss and potential humidifier contamination. The vibrating mesh nebulizer and the jet nebulizer were placed on the dry side. METHODS: Aerosol delivery was measured using multiple ventilator settings (inspiratory time $=\mathbf{0 . 4 5}-1.01 \mathrm{~s}$ ). Using radiolabeled saline and a gamma camera, bench studies were performed using a ventilator to test 4 breathing patterns. Four scenarios were assessed during testing: $3 \mathrm{~mL}$ and $6 \mathrm{~mL}$ fill volumes with and without heated wire humidification. Measurements included inhaled mass (as a percentage of the nebulizer charge), nebulizer residual, mass balance, and aerosol particle size distribution. Statistics were determined using MannWhitney and linear regression. RESULTS: The inhaled mass for the breath-enhanced jet nebulizer was $10.5-29.2 \%$ and was affected by fill volume $(P=.004)$ but not by humidity. The inhaled mass for the vibrating mesh nebulizer was $0.9-33 \%$ and was unaffected by fill volume and humidity. The inhaled mass for the jet nebulizer was $\mathbf{2 . 5}-\mathbf{2 5 . 9 \%}$ and was affected by both fill volume $(P=.009)$ and humidity $(3 \mathrm{~mL}, P=.002)$. The inhaled mass for the vibrating mesh nebulizer was more variable due to random failures to achieve complete nebulization, and inhaled mass correlated closely with residual mass: IM\% $=-0.233($ Residual $\%)+24.3, r^{2}=0.67, P<.001$. For all devices, large particles were lost in the ventilator tubing; large particles were also lost in the humidifier for the vibrating mesh nebulizer (17\% nebulizer charge), resulting in similar particle distributions (mass median aerodynamic diameter 1.33-1.95 $\mu \mathrm{m}$ ) for all devices. CONCLUSIONS: Nebulization with the breathenhanced jet nebulizer was less sensitive to humidification than the jet nebulizer. Delivery via the vibrating mesh nebulizer was not predictable, with random failure to empty (55\% experimental runs). All devices delivered similar particle distributions. Wet-side aerosol delivery avoids humidifier contamination, and breath-enhanced technology can ensure better control of drug delivery. Key words: aerosols; nebulizers and vaporizers; administration; inhalation; ventilators; mechanical; humidifiers; drug delivery; breath-enhanced. [Respir Care 2020;65(10):1419-1426. (C) 2020 Daedalus Enterprises]
\end{abstract}

\section{Introduction}

Researchers have been studying aerosol delivery during mechanical ventilation for more than 30 years, yet there is no standard method for aerosol therapy. Uncontrolled variables include ventilator effects, such as bias flow, ${ }^{1,2}$

Dr Ashraf, Dr Smaldone, and Mr McPeck are affiliated with the Division of Pulmonary, Critical Care and Sleep Medicine, Department of Medicine, Stony Brook University Medical Center, Stony Brook, New York. Ms Cuccia is affiliated with the State University of New York at Stony Brook, Department of Respiratory Care, Stony Brook, New York. breath actuation settings, ${ }^{2-4}$ methods of humidification,,${ }^{2,5-8}$ nebulizer positioning, ${ }^{1,5,9-11}$ nebulizer technology, ${ }^{5-7,11,12}$ and device reproducibility. ${ }^{13}$ Many in vitro studies have tested these variables following ad hoc protocols, leading to an evolution of delivery techniques, especially changes

\footnotetext{
A version of this paper was presented at the OPEN Forum of the AARC Congress 2019, held November 9-12, 2019, in New Orleans, Louisiana.

This study was supported in part by InspiRx (Somerset, New Jersey). Dr Smaldone and Ms Cuccia have disclosed relationships with InspiRx Dr Ashraf and Mr McPeck disclosed no conflicts of interest.
} 
in ventilators, their circuits, methods of humidification, and nebulizer technologies.

\section{See the Related Editorial on Page 1624}

The present study was designed to assess current technologies over a range of practical settings and situations to provide a modern understanding of aerosol delivery during mechanical ventilation. Specifically, the protocol tested available technologies in terms of inter- and intradevice variability and sensitivity to humidification and volume-fill effects under real-use conditions. Three nebulizer technologies were tested: a novel breath-enhanced jet nebulizer called the i-AIRE (InspiRx, Somerset, New Jersey); a vibrating mesh nebulizer (Solo, Aerogen, Galway Ireland); and a jet nebulizer (Hudson MicroMist, Teleflex Medical, Morrisville, North Carolina). Nebulizer placement in the circuit was based on current practices which favor nebulizer location proximal to the ventilator. ${ }^{1,5,6,9,14,15}$ This position facilitates control of device orientation which may affect function. Effects of contemporary humidification systems were tested with each nebulizer technology over a range of ventilator settings and fill volumes to measure their performance and reliability. The vibrating mesh nebulizer and the jet nebulizer were located on the dry side of the humidifier ${ }^{1,5,15}$ (ie, on the humidifier inlet), whereas the breath-enhanced jet nebulizer was located on the wet side (ie, on the humidifier outlet). This study was performed at the Aerosol Laboratory in the Division of Pulmonary, Critical Care, and Sleep Medicine, in the Department of Medicine at Stony Brook University Medical Center, Stony Brook, New York.

\section{Methods}

\section{Experimental Setup}

The experimental setups are illustrated in Figure 1 (humidified) and Figure 2 (nonhumidified). In Figure 1, the breathenhanced jet nebulizer is shown in the circuit located on the wet side (outlet) of a conventional heated humidifier (ConchaTherm Neptune, Hudson RCI/Teleflex Medical, Morrisville North Carolina) set at $37^{\circ} \mathrm{C}$. This position has been found to be more effective for this type of nebulizer technology. ${ }^{16}$ During treatment, the 2-way selector valve was turned, directing all inspiratory gas flow from the ventilator to flow through the top of the nebulizer and exit into the inspiratory limb of the circuit through the side port, thereby enhancing aerosol generation primarily during inspiration. ${ }^{16}$ Wall air at a

Correspondence: Sunya Ashraf MD. E-mail: sunya.ashraf@stonybrook medicine.edu.

DOI: $10.4187 /$ respcare.07639

\section{QUICK LOOK}

\section{Current knowledge}

During invasive ventilation, vibrating mesh nebulizers with low residual mass are reported to be more efficient than conventional jet nebulizers. Breath-enhanced jet nebulization is new for mechanical ventilation and has not been compared to existing technology. In addition, how these devices interact with other factors potentially affecting drug delivery (eg, active humidification, nebulizer fill volume, and day-to-day inter- and intradevice usage) is unknown.

\section{What this paper contributes to our knowledge}

Although vibrating mesh nebulizers may be highly efficient, they were unpredictable and widely variable in output. Conventional jet nebulizers were sensitive to fill volume and humidification, consistent with previously published studies. Using the breath-enhanced jet nebulizer on the wet side of the heated humidifier during mechanical ventilation can provide predictable and reliable drug delivery while avoiding effects of active humidification and humidifier contamination.

flow of $3.5 \mathrm{~L} / \mathrm{min}$ at 50 psi was turned on to power the nebulizer. The humidifier outlet temperature sensor regulating the humidifier was relocated from the standard location on the proximal end of the conventional inspiratory limb to the point where the 2-way selector valve was placed on the humidifier outlet. The circuit is designed so that when a treatment is completed, the gas flow powering the nebulizer is turned off and the selector valve is turned, bypassing the nebulizer and directing ventilator flow to the inspiratory limb of the circuit. The inset diagrams on Figure 1 depict the Aerogen vibrating mesh nebulizer and the Hudson jet nebulizer configurations, both placed on the dry side of the humidifier as recommended. ${ }^{15,17}$

Figure 2 shows the i-AIRE breath-enhanced jet nebulizer in the nonhumidified experimental setup. Although a heat-andmoisture exchanger (HME) was not used during this study, an aerosol bypass HME is shown to demonstrate its mounting location if used clinically. In this configuration, the breathenhanced jet nebulizer, as well as the vibrating mesh nebulizer and jet nebulizer (shown in the inset diagram), were placed on the inspiratory limb $\sim 15 \mathrm{~cm}(\sim 6$ inches) from the ventilator outlet, which has been described as an optimal position. ${ }^{1,5}$ The Hudson MicroMist jet nebulizer was attached to the ventilator circuit with a Hudson spring-loaded T-adapter and operated at a flow of $8 \mathrm{~L} / \mathrm{min}$ as per manufacturer instructions using wall air at 50 psi. The Aerogen Solo vibrating mesh nebulizer was positioned in the circuit using the adult Aerogen T-adapter as per manufacturer specifications ${ }^{15}$ and operated via an Aerogen Pro-X Controller. 


\section{Nebulization During Mechanical Ventilation}
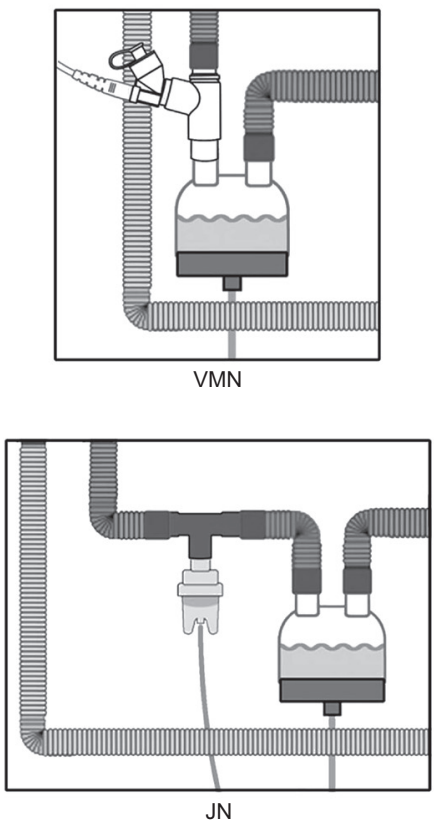

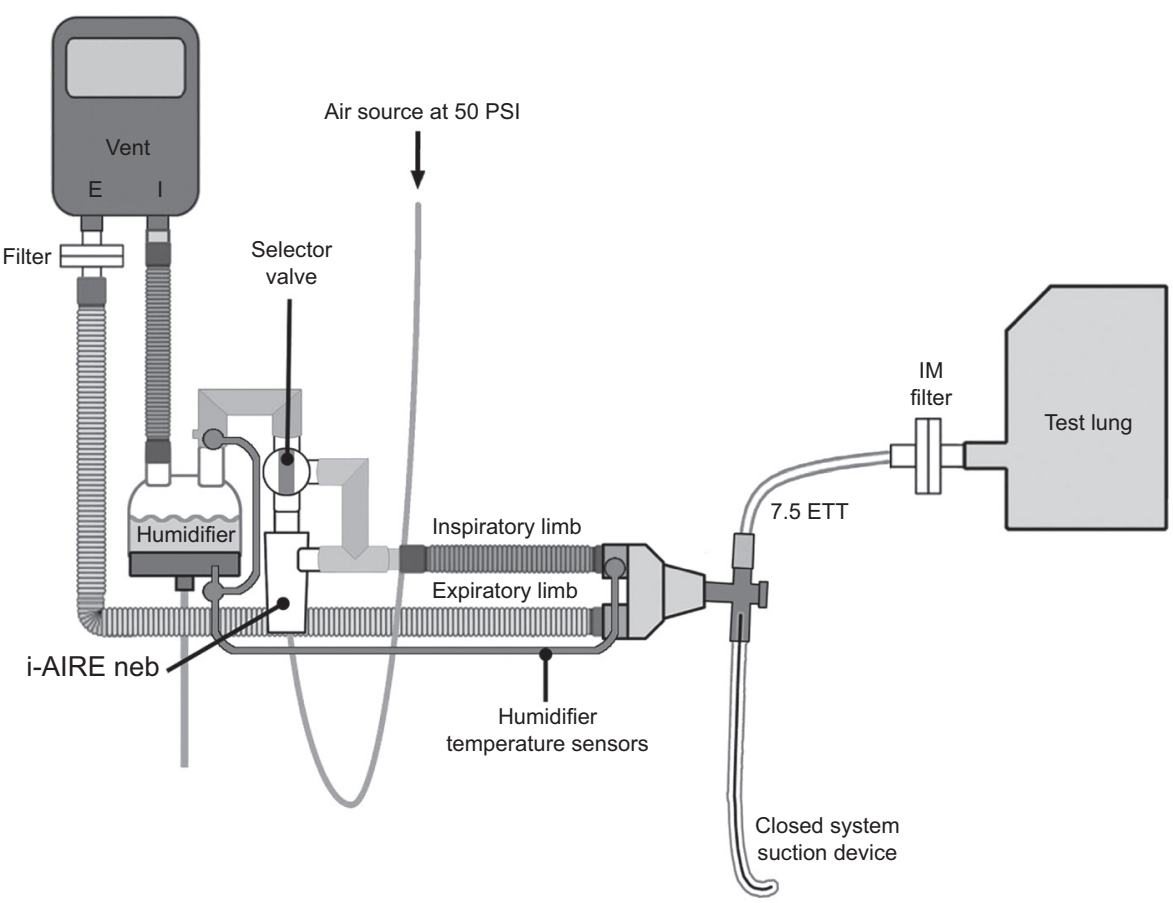

Fig. 1. Location of the i-AIRE breath-enhanced jet nebulizer connected to the heated humidifier at its outlet port (ie, the wet side) during active humidification. The selector valve may be turned so that inspiratory gas from the ventilator either bypasses the nebulizer or passes through it, for breath-enhancement, as gas flows to the patient. The inset diagrams show the vibrating mesh nebulizer and the jet nebulizer connected to the heated humidifier at its inlet port (ie, the dry side). VMN = vibrating mesh nebulizer; $\mathrm{JN}=$ jet nebulizer.
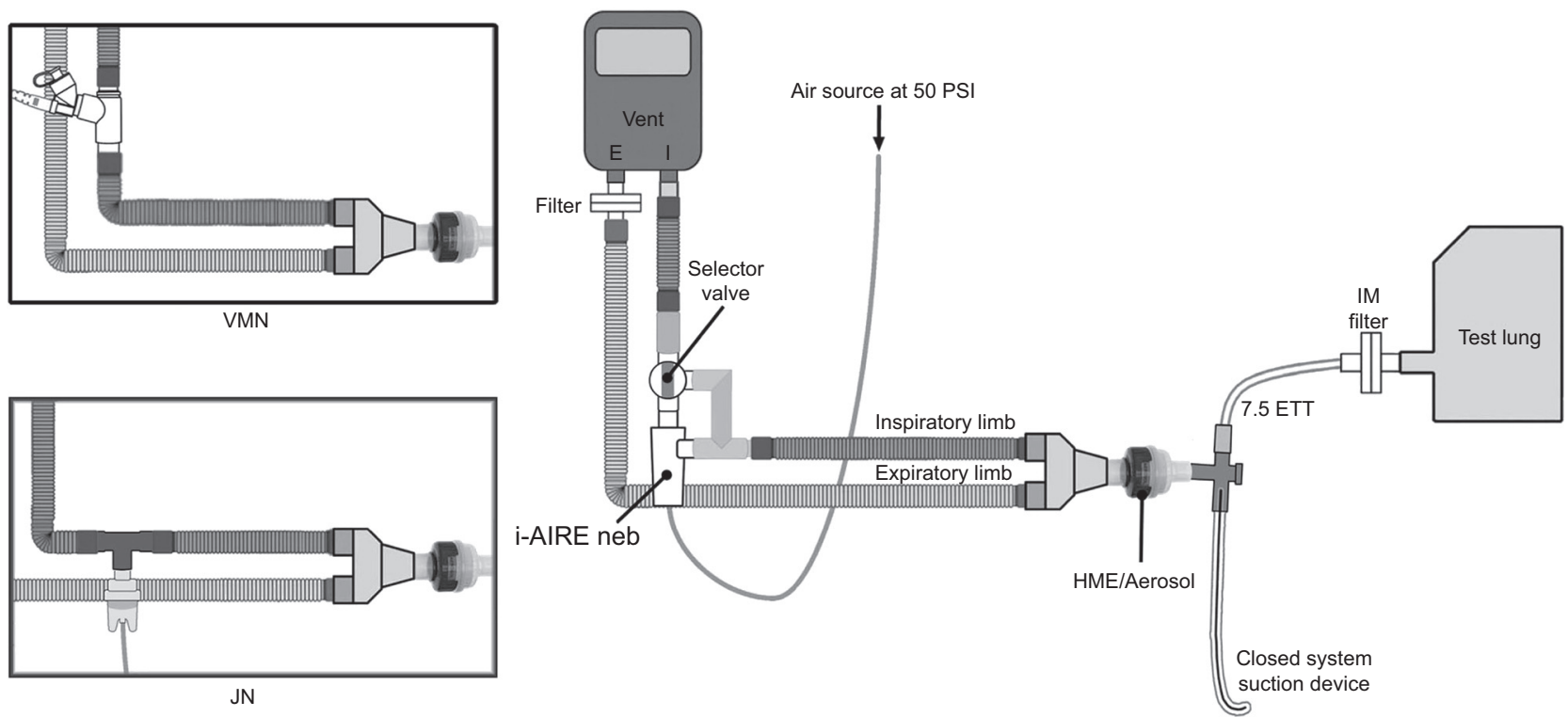

Fig. 2. Although a heat-and-moisture exchanger (HME) was not used during this study, an aerosol bypass HME is shown to demonstrate its mounting location if used clinically. All nebulizers were placed $\sim 15 \mathrm{~cm}(\sim 6$ inches) from the ventilator inspiratory limb outlet port. The i-AIRE breathenhanced jet nebulizer with its selector valve was interposed between the ventilator outlet port and the inspiratory limb of the circuit. The inset diagrams show the vibrating mesh nebulizer and the jet nebulizer interposed into the inspiratory limb with their respective T-adapters. IM $=$ inhaled mass; $\mathrm{ETT}=$ endotracheal tube; $\mathrm{VMN}=$ vibrating mesh nebulizer; $\mathrm{JN}=$ jet nebulizer.

To duplicate typical hospital ventilator circuit setups, the patient $\mathrm{Y}$-connector was attached to a closed-suction system device (Ballard Closed Suction System, Avanos
Medical, Alpharetta, Georgia) and a 7.5-mm inner diameter endotracheal tube (Rusch, Teleflex Medical). The circuit was connected to a Training Test Lung (Michigan 
Table 1. Ventilator Settings

\begin{tabular}{|c|c|c|c|c|c|}
\hline Mode & $\mathrm{f}$, breaths/min & $\mathrm{V}_{\mathrm{T}} /$ Pressure Control & Inspiratory Time, $\mathrm{s}$ & Total Cycle Time, s & Duty Cycle \\
\hline VC-CMV & 18 & $500 \mathrm{~mL}$ & 0.7 & 3.33 & 0.21 \\
\hline VC-CMV & 15 & $460 \mathrm{~mL}$ & 0.5 & 4 & 0.13 \\
\hline VC-CMV & 20 & $650 \mathrm{~mL}$ & 1.01 & 3 & 0.34 \\
\hline PC-CMV & 18 & $15 \mathrm{~cm} \mathrm{H}_{2} \mathrm{O}$ & 0.8 & 3.33 & 0.24 \\
\hline
\end{tabular}

* Duty cycle $=$ inspiratory time/total time cycle.

$\mathrm{f}=$ frequency; $\mathrm{V}_{\mathrm{T}}=$ tidal volume; $\mathrm{VC}-\mathrm{CMV}=$ volume control continuous mandatory ventilation; $\mathrm{PC}-\mathrm{CMV}=$ pressure control continuous mandatory ventilation

Instruments, Grand Rapids, Michigan) set with a resistance of $5 \mathrm{~cm} \mathrm{H}_{2} \mathrm{O}$ and compliance of $40 \mathrm{~mL} / \mathrm{cm} \mathrm{H}_{2} \mathrm{O}$. The Avea ventilator (Vyaire Medical, Mettawa, Illinois) was used with 4 different clinically relevant breathing patterns (Table 1) chosen to provide a range of ventilator duty cycles: 3 volume control modes with tidal volumes of $460-650 \mathrm{~mL}$, and 1 pressure control mode with a pressure limit of $15 \mathrm{~cm} \mathrm{H}_{2} \mathrm{O}$. Frequencies of 15-20 breaths/min, PEEP of $5 \mathrm{~cm} \mathrm{H}_{2} \mathrm{O}$, and bias flow of $2.0 \mathrm{~L} / \mathrm{min}$ at $21 \%$ oxygen was used for all settings. Nebulizers were operated continuously and were initially run to dryness (ie, cessation of visible aerosol output) using visual inspection. Run time was measured using a stopwatch.

\section{Inhaled Mass and Residual Activity}

An inhaled mass (IM) filter (Pari, Starnberg, Germany) placed at the distal tip of the endotracheal tube collected the aerosol particles that would be inhaled by a patient under similar conditions. A similar filter was placed in the expiratory limb proximal to the exhalation channel for mass balance measurements. A total of 58 experiments were performed using 4 new devices of each nebulizer type. All nebulizers were rotated throughout the experimental protocol and tested a minimum of 4 times each for repeatability. Some nebulizers were tested more frequently because they were used for mass balance experiments. Each nebulizer was charged with either 3 $\mathrm{mL}$ or $6 \mathrm{~mL}$ of Technetium-99m radiolabeled saline. For all experiments, radioactivity placed in the nebulizers and radioactivity captured on the filters were measured with a gamma camera (Maxi Camera 400, General Electric, Horsholm, Denmark; Power Computing, Model 604/150/D, Austin, Texas; Nuclear Mac OS 4.2.2, Scientific Imaging, Thousand Oaks, California). At the conclusion of test runs, nebulizers were placed on the gamma camera to determine residual radioactivity, expressed as percent of nebulizer charge (Residual\%).

\section{Mass Balance}

In a separate set of experiments, for each experimental configuration depicted in Figure 1 and Figure 2, a complete mass balance determination was carried out for each nebulizer type for the 3-mL fill volume. The radioactivity of aerosol deposited on circuit components was measured by placing each component separately on the gamma camera.

\section{Aerosol Particle Size Distribution}

Aerosol particle size distribution was determined via cascade impaction sampling at the distal tip of the endotracheal tube (Marple 8-stage impactor, Thermo Fischer Scientific, Waltham, Massachusetts) with a vacuum flow of $2.5 \mathrm{~L} / \mathrm{min}$. Distributions were determined for 2 samples of each nebulizer type using a 3-mL fill volume. Aerosols were sampled over a 3-min period. Radioactivity on the cascade stages was measured with a collimated ratemeter (Ludlum Measurements, Sweetwater, Texas), and the distribution was plotted on log probability paper to determine mass median aerodynamic diameter. ${ }^{18}$

\section{Statistical Analysis}

GraphPad Prism 8.3 for Mac OS (GraphPad Software, San Diego, California) was used to calculate mean \pm SD for each nebulizer type with and without humidification and to generate log particle size versus probability graphs for aerosol particle size distribution. The Mann-Whitney test and linear regression analysis were used to assess inter- and intradevice variability. A $P$ value $<.05$ was considered statistically significant.

\section{Results}

Aerosol delivery to the filter for each device, expressed as IM as a percent of nebulizer charge (IM\%), is shown in Figure 3. Measurements of IM\% for each experiment were plotted with IM\% on the $y$ axis against inspiratory time $\left(\mathrm{T}_{\mathrm{I}}\right)$ on the $x$ axis, and different symbols were used to indicate humidification status and fill volume. Each data point represents 1 of the 4 devices for each nebulizer type as a function of ventilator setting (expressed as $\mathrm{T}_{\mathrm{I}}$, a common variable for all settings), humidification, and fill volume. Statistical data for each configuration is tabulated in 


\section{Nebulization During Mechanical Ventilation}
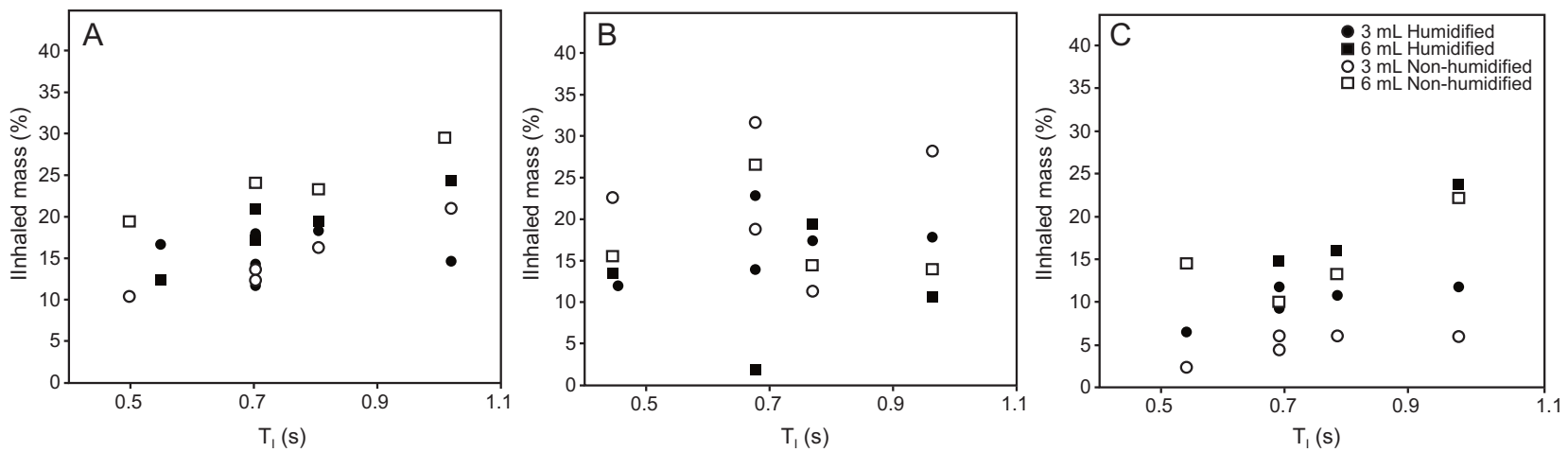

Fig. 3. Inhaled mass versus inspiratory time $\left(T_{1}\right)$ for $A$ : breath-enhanced nebulizer, $B$ : vibrating mesh nebulizer, and C: jet nebulizer.

Table 2. Inhaled Mass Effects of Humidification and Fill Volume

\begin{tabular}{|c|c|c|c|c|c|c|c|c|c|c|c|c|}
\hline & $3 \mathrm{~mL}$ Wet & 3 mL Dry & $P$ & $6 \mathrm{~mL} \mathrm{Wet}$ & $6 \mathrm{~mL}$ Dry & $P$ & $3 \mathrm{~mL} \mathrm{Wet}$ & $6 \mathrm{~mL} \mathrm{Wet}$ & $P$ & 3 mL Dry & 6 mL Dry & $P$ \\
\hline \multicolumn{13}{|c|}{ Breath-enhanced jet nebulizer } \\
\hline No. & 7 & 6 & & 4 & 5 & & 7 & 4 & & 6 & 5 & \\
\hline Mean \pm SD & $12.2 \pm 6.2$ & $15.6 \pm 2.5$ & .18 & $23.9 \pm 4$ & $18.8 \pm 4.3$ & .21 & $12.2 \pm 6.2$ & $23.9 \pm 4$ & .01 & $15.6 \pm 2.5$ & $18.8 \pm 4.3$ & .18 \\
\hline \multicolumn{13}{|c|}{ Vibrating mesh nebulizer } \\
\hline No. & 5 & 5 & & 4 & 4 & & 5 & 4 & & 5 & 4 & \\
\hline Mean \pm SD & $23.1 \pm 8.6$ & $17 \pm 4.5$ & .31 & $17.9 \pm 6.5$ & $11.1 \pm 7.9$ & .2 & $23.1 \pm 8.6$ & $17.9 \pm 6.5$ & .41 & $17 \pm 4.5$ & $11.1 \pm 7.9$ & .29 \\
\hline \multicolumn{13}{|l|}{ Jet nebulizer } \\
\hline No. & 6 & 5 & & 4 & 4 & & 6 & 4 & & 5 & 4 & \\
\hline Mean \pm SD & $5.5 \pm 1.6$ & $10.9 \pm 2.4$ & .002 & $16.3 \pm 5.7$ & $18.8 \pm 4.8$ & .2 & $5.5 \pm 1.6$ & $16.3 \pm 5.7$ & .009 & $10.9 \pm 2.4$ & $18.8 \pm 4.8$ & .008 \\
\hline
\end{tabular}

Table 3. Mass Balance-3 mL Fill Volume

\begin{tabular}{|c|c|c|c|c|c|c|}
\hline \multirow{2}{*}{ Mass Balance } & \multicolumn{2}{|c|}{ Breath-Enhanced Jet Nebulizer } & \multicolumn{2}{|c|}{ Vibrating Mesh Nebulizer } & \multicolumn{2}{|c|}{ Jet Nebulizer } \\
\hline & Humidified & HME & Humidified & HME & Humidified & HME \\
\hline Residual & 27.6 & 42.8 & 6.1 & 6.9 & 69.9 & 63.8 \\
\hline Inhaled mass filter & 13.8 & 11.8 & 19.1 & 23.5 & 6.5 & 12.8 \\
\hline Expiratory limb filter & 18.7 & 14.1 & 16.7 & 26.2 & 7.0 & 6.2 \\
\hline T-adapter & 2.2 & 2.8 & 9.3 & 6.4 & 6.1 & 6.4 \\
\hline Endotracheal tube & 6.3 & 2.8 & 6.6 & 1.1 & 1.3 & 1.1 \\
\hline Closed system suction & 0.4 & 0.3 & 1.1 & 0.4 & 0.3 & 0.4 \\
\hline Circuit & 23.3 & 20.8 & 26 & 42.8 & 5.1 & 6.2 \\
\hline Humidifier & 0 & N/A & 17.1 & N/A & .1 & N/A \\
\hline Total & 94.1 & 95.4 & 102 & 106.9 & 96.3 & 96.9 \\
\hline
\end{tabular}

Table 2. Figure 3 shows that, for both the breath-enhanced jet nebulizer and the jet nebulizer, there was a gradual increase in delivery with increasing $\mathrm{T}_{\mathrm{I}}$, which suggests some sensitivity to increasing duty cycle. The vibrating mesh nebulizer's delivery did not appear to be affected by $\mathrm{T}_{\mathrm{I}}$ with more scatter over the range of ventilator settings. Mass balance data are tabulated in Table 3 .

\section{Breath-Enhanced Jet Nebulizer}

IM\% was significantly affected by fill volume, but only when humidified: $12.2 \pm 6.2 \%$ for $3-\mathrm{mL}$ fill volume and $23.9 \pm 4.0 \%$ for 6 - $\mathrm{mL}$ fill volume $(P=.004)$. Humidifcation effects were not significant. For the 3 -mL volume fill, the mass balance determination indicated that the nebulizer 


\section{Nebulization During Mechanical Ventilation}

Residual\% was decreased (Table 3) and circuit losses were higher with humidification, resulting in small insignificant changes in IM\% with humidity. Mean run times for the 3$\mathrm{mL}$ and 6-mL fill volume were 8 and $18 \mathrm{~min}$, respectively.

\section{Vibrating Mesh Nebulizer}

Humidity and fill volume had no significant effects on IM\%. Visually, however, data for the vibrating mesh nebulizer varied over a wider range than the other devices (Fig. 3). Some of this variability was related to variables intrinsic to the nebulizer (ie, separate from the conditions of the ventilator circuit). The factors influencing vibrating mesh nebulizer function were assessed in Figure 4, which is a plot of IM\% against nebulizer Residual\%. Some devices emptied nearly completely with Residual\% being $<10 \%$ (left side of Fig. 4), close to the small Residual\% expected for this technology. However, for all the data, variation in IM\% was closely

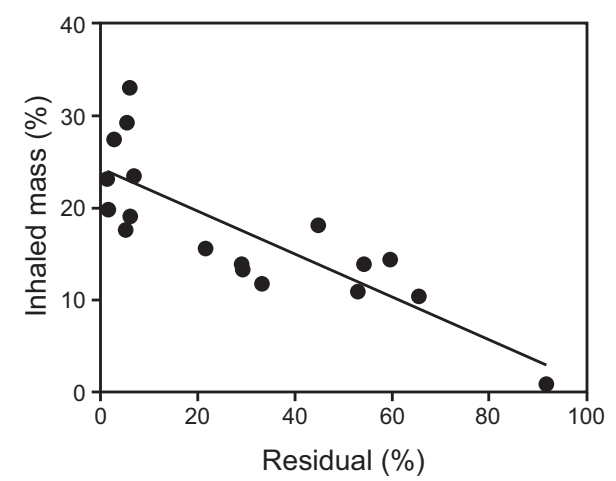

Fig. 4. Inhaled mass versus nebulizer residual for vibrating mesh nebulizer experiments: inhaled mass $=-0.233$ (Residual\%) +24.3 , $r^{2}=50.67, P<.001$ correlated with wide variation in the nebulizer Residual\%. Overall, the Solo failed to empty $55 \%$ of the time (ie, 10 of 18 runs). Failure to empty occurred randomly; for example, a device would empty completely on one run and fail to empty on the next run. When the nebulizer functioned properly (ie, with a low Residual\%), the mass balance determination (Table 3) demonstrated a loss of $17 \%$ in the humidifier during humidification and $26 \%$ in the vent circuit. In the nonhumidified circuit, $48.6 \%$ was lost. The average run times for $3-\mathrm{mL}$ and 6 $\mathrm{mL}$ fill volumes were $11 \mathrm{~min}$ and $20 \mathrm{~min}$, respectively.

\section{Jet Nebulizer}

IM\% was strongly affected by fill volume, increasing 2-fold regardless of humidity: $5.5 \pm 1.6 \%$ for $3 \mathrm{~mL}$ and $16.3 \pm 5.7 \%$ for $6 \mathrm{~mL}(P=.009)$ when actively humidified versus $10.9 \pm 2.4 \%$ for $3 \mathrm{~mL}$ and $18.8 \pm 8.8 \%$ for $6 \mathrm{~mL}$ for nonhumidified $(P=.007)$. For a given fill volume, significant humidification effects were seen only for the $3-\mathrm{mL}$ fill volume: $5.5 \pm 1.6 \%$ humidified compared to $10.9 \pm 2.6 \%$ for nonhumidified $(P=.002)$. The mass balance determination (Table 3 ) indicated minute losses in the humidifier of $0.1 \%$, with nebulizer Residual $\%$ as high as $70 \%$. Average run times for $3-\mathrm{mL}$ and 6-mL fill volumes were 6 and $17 \mathrm{~min}$, respectively.

\section{Aerosol Particle Size Distribution}

Aerosol particle size distributions at the distal tip of the endotracheal tube were similar for all 3 devices (Figure 5). The mean \pm SD of 2 samples of each nebulizer type, with a 3 -mL fill volume, with and without humidification, were $1.95 \pm 0.21 \mu \mathrm{m}$ for the humidified breath-enhanced jet nebulizer; $1.45 \pm 0.01 \mu \mathrm{m}$ for the nonhumidified breathenhanced jet nebulizer; $1.90 \pm 0.14 \mu \mathrm{m}$ for the humidified vibrating mesh nebulizer; $1.57 \pm 0.05 \mu \mathrm{m}$ for the
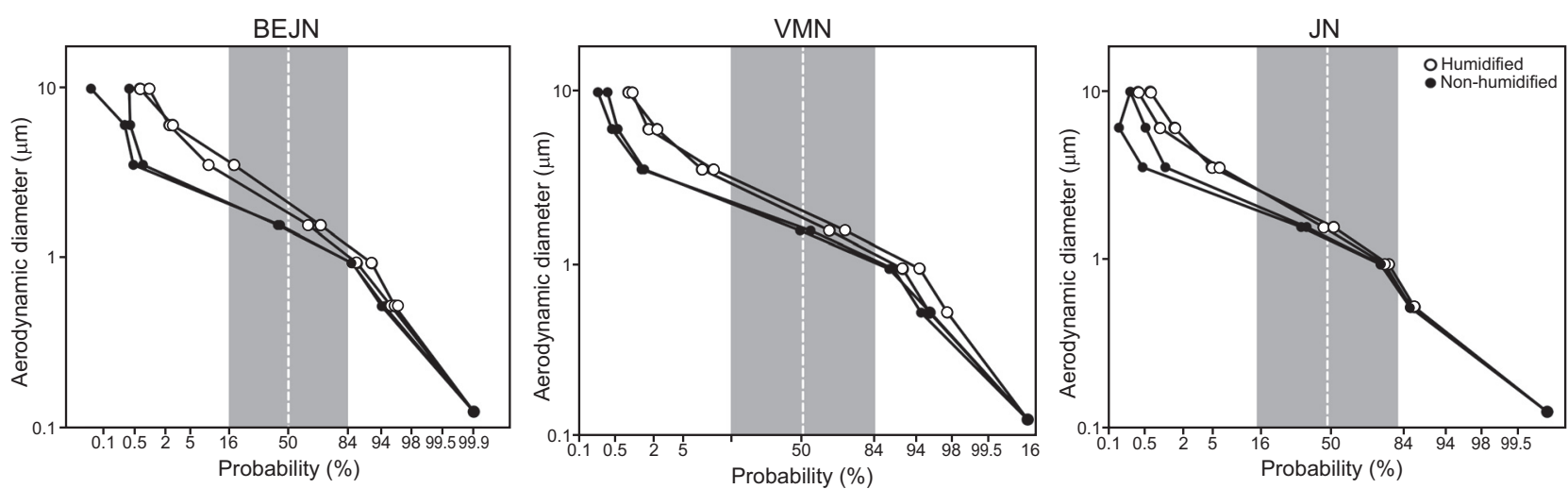

Fig. 5. Aerosol particle size distributions for 3 nebulizer types, with and without heated humidification. The test breathing pattern consisted of volume control continuous mechanical ventilation, frequency $=18$ breaths $/ \mathrm{min}$, tidal volume $=500 \mathrm{~mL}$, inspiratory flow $=45 \mathrm{~L} / \mathrm{min}$, inspiratory time $=$ $0.7 \mathrm{~s}, \mathrm{PEEP}=5 \mathrm{~cm} \mathrm{H} \mathrm{H}_{2} \mathrm{O}$, and bias flow $\left.=2.0 \mathrm{~L} / \mathrm{min}\right)$. Log particle size for each cascade stage plotted against probability. The following values are presented as mean \pm SD at 50\% probability: mass median aerodynamic diameter: breath-enhanced jet nebulizer $=1.95 \pm 0.21 \mu \mathrm{m}$ (humidified), $1.45 \pm 0.01 \mu \mathrm{m}$ (nonhumidified); vibrating mesh nebulizer $=1.90 \pm 0.14 \mu \mathrm{m}$ (humidified), $1.57 \pm 0.05 \mu \mathrm{m}$ (nonhumidified); jet nebulizer $=1.55 \pm$ $0.07 \mu \mathrm{m}$ (humidified), $1.33 \pm 0.03 \mu \mathrm{m}$ (nonhumidified). 


\section{Nebulization During Mechanical Ventilation}

nonhumidified vibrating mesh nebulizer; $1.55 \pm 0.07 \mu \mathrm{m}$ for the humidified jet nebulizer; and $1.33 \pm 0.03 \mu \mathrm{m}$ for the nonhumidified jet nebulizer.

\section{Discussion}

Aerosol delivery to the ventilated patient is continuously evolving. In this study, we tested representative samples of 3 current nebulizer technologies over a range of settings with and without humidification using fill volumes typical for common drugs. We tested multiple examples of each device to mimic real-world usage, as we sought to document both inter- and intradevice variability. Common drugs such as bronchodilators that are used off-label on ventilators were originally approved for hand-held nebulization. Their clinical effectiveness is often monitored at the bedside because the dose response of these devices cannot be predicted for the individual patient. It is reasonable to assume that knowledge of a device's performance characteristics will enhance the caregiver's assessment of a therapy's effectiveness. The jet nebulizers tested in this study were powered from wall air to control delivery conditions because modern ventilators do not have standardized jet nebulizer driving systems, and in some cases they do not support jet nebulization at all.

On average, the breath-enhanced jet nebulizers and the vibrating mesh nebulizers demonstrated equal overall delivery, with both devices more efficient than the conventional jet nebulizers. Vibrating mesh nebulizer delivery, however, was less predictable, with delivery ranging between $0.9 \%$ and $33 \%$ in individual experiments. The vibrating mesh nebulizer behaved differently than the jet nebulizer devices, exhibiting greater variation in IM\%. Figure 4 displays the relationship between the IM\% and Residual\%. The residual mass for each run was expected to be a low percentage (ie, $<10 \%$ ). There were 2 populations of Residual\% values: one group was clustered at low values, and the other was randomly elevated. The former represents intrinsic differences between the devices, perhaps due to membrane function or other uncontrolled factors; the latter represents failure of the device to empty. Because each example of the Solo tested was rotated between experiments, the failure to empty was not a hard failure, that is, not an electrical or mesh issue, rather a random failure to empty. The same device that stopped nebulizing on a given run functioned normally on a repeat run. This observation is not a mesh defect but is likely related to failure of the liquid stream from the reservoir to contact the mesh. This behavior has been described previously for isolated devices tested during continuous nebulization with fill volumes of $3 \mathrm{~mL} \cdot{ }^{13}$ For the jet nebulizers in this study, the Residual\% was relatively reproducible for each condition.

There were important differences between devices when used with humidification. Aerosol delivery for the newer technologies was less sensitive to humidification. Effects on vibrating mesh nebulizer aerosol delivery were not detectable, although the dependence of delivery on device emptying may have obscured potential effects of humidification. For the breath-enhanced jet nebulizer, compared to nonhumidified delivery, wet side nebulization had a lower Residual\%. This unique observation was first reported for this technology by Cuccia et al, ${ }^{16}$ who studied a breathactuated version of the i-AIRE breath-enhanced jet nebulizer. Contrary to conventional jet nebulization, where expected reductions in aerosol delivery can approach 50\%, ${ }^{6}$ Cuccia et al ${ }^{16}$ reported that wet side nebulization with the i-AIRE resulted in preserved overall delivery with humidification. In both the study by Cuccia et $\mathrm{al}^{16}$ and the present study, the decreased Residual\% was matched by increased losses in the ventilator tubing resulting in similar aerosol delivery between dry and wet circuits (Table 3 ).

Cuccia et $\mathrm{al}^{16}$ used the $\mathrm{i}$-AIRE in a breath-actuated mode in which the $3.5-\mathrm{L} / \mathrm{min}$ air flow powering the nebulizer was interrupted during expiration by a computer-driven solenoid valve. Under those conditions, the authors reported an IM\% of $31.1 \pm 6.33 \%$, approximately double that seen in our results. In other words, their breath-actuated device avoided aerosol losses during expiration. In this study, the i-AIRE in continuous operation (without the solenoid trigger) is compared with other devices currently in use for routine aerosol therapy (eg, bronchodilators).

The conventional jet nebulizer behaved as expected for this technology. ${ }^{2}$ Specifically, IM\% was significantly reduced with humidity at $3 \mathrm{~mL}$ volume fill. This effect was first reported on the bench in 1992 by O'Riordan et al, ${ }^{6}$ with in vivo confirmation by Miller et al, ${ }^{2}$ who reported that active humidification significantly reduced delivery of inhaled antibiotics to intubated patients. Those data led Palmer and colleagues ${ }^{19-21}$ to design clinical protocols to test effects of inhaled antibiotics delivered while deliberately avoiding circuit humidification during active nebulization. Our results indicate that wet side nebulization with the i-AIRE breathenhanced jet nebulizer avoids the adverse effects on aerosol delivery and ensure that clinicians can expect equal efficiency of delivery with or without active humidification. Both the iAIRE breath-enhanced jet nebulizer and the Hudson jet nebulizer were sensitive to changes in nebulizer fill volume, showing significant increases in efficiency with increased volume. For the Hudson jet nebulizer, the Residual\% was the highest of all tested devices, and delivery was relatively low under all conditions unless the nebulizer charge was increased to $6 \mathrm{~mL}$. The increase in IM\% with a higher fill volume is a known factor for jet nebulizers during spontaneous breathing; the higher the fill volume, the greater the output. ${ }^{2,12,22,23}$ Overall, the Aerogen vibrating mesh nebulizer was most efficient with the lowest Residual\%, but those gains were largely canceled by repeated failure of the vibrating mesh nebulizer to empty. ${ }^{13}$

In spite of its dry-side location, the Hudson jet nebulizer did not deposit particles in the humidifier, whereas the 


\section{Nebulization During Mechanical Ventilation}

Aerogen vibrating mesh nebulizer deposited $17 \%$ of the dose. The differences in behavior between jet and mesh devices may be due to the $8 \mathrm{~L} / \mathrm{min}$ of dry air flow used to drive the jet nebulizer. The high gas flow for the jet nebulizer may affect local particle size such that the largest particles are immediately reduced in size, thus allowing aerosol to pass through the humidifier without depositing.

Respiratory therapists are aware that expiratory volume readouts on the ventilator are commonly affected during jet nebulization. However, from previous studies in our laboratory, jet flow ranging from 3.5 to $10 \mathrm{~L} / \mathrm{min}$ had minimal effect on delivered volume. ${ }^{16,24}$

Finally, our data indicate that, independent of the technology used, the aerosol particle size distributions measured at the distal tip of the endotracheal tube were similar for all 3 devices, with a mass median aerodynamic diameter of 1.33-1.95 $\mu \mathrm{m}$, demonstrating that large particles are lost in the circuit.

\section{Conclusions}

When nebulizing medication during conventional mechanical ventilation, there are important differences in drug delivery between commercially available delivery devices that are independent of the ventilator. Knowledge of these differences could account for clinical differences in response.

\section{ACKNOWLEDGMENTS}

The authors thank Lorraine Morra for her time and assistance with technical and statistical analysis. We also appreciate the loan of equipment and supplies from the Respiratory Care Department at Stony Brook University.

\section{REFERENCES}

1. Ari A, Atalay OT, Harwood R, Sheard MM, Aljamhan EA, Fink JB. Influence of nebulizer type, position, and bias flow on aerosol drug delivery in simulated pediatric and adult lung models during mechanical ventilation. Respir Care 2010;55(7):845-851.

2. Miller DD, Amin MM, Palmer LB, Shah AR, Smaldone GC. Aerosol delivery and modern mechanical ventilation: in vitro/in vivo evaluation. Am J Respir Crit Care Med 2003;168(10):1205-1209.

3. Ehrmann S, Lyazidi A, Louis B, Isabey D, Le Pennec D, Brochard L, et al. Ventilator-integrated jet nebulization systems: tidal volume control and efficiency of synchronization. Respir Care 2014;59(10):1508-1516.

4. McPeck M, O'Riordan T, Smaldone GC. Choice of mechanical ventilator: influence on nebulizer performance. Respir Care 1993;38(8):887-895.

5. Ari A, Areabi H, Fink JB. Evaluation of aerosol generator devices at 3 locations in humidified and non-humidified circuits during adult mechanical ventilation. Respir Care 2010;55(7):837-844.
6. O'Riordan TG, Greco MJ, Perry RJ, Smaldone GC. Nebulizer function during mechanical ventilation. Am Rev Respir Dis 1992;145(5):1117-1122.

7. O'Riordan TG, Palmer LB, Smaldone GC. Aerosol deposition in mechanically ventilated patients: optimizing nebulizer delivery. Am J Respir Crit Care Med 1994;149(1):214-219.

8. Diot P, Morra L, Smaldone GC. Albuterol delivery in a model of mechanical ventilation: comparison of metered-dose inhaler and nebulizer efficiency. Am J Respir Crit Care Med 1995;152(4 Pt 1):1391-1394

9. Hughes J, Saez T. Effects of nebulizer mode and position in a mechanical ventilator circuit on dose efficiency. Respir Care 1987;32(12): 1131-1135.

10. Berlinski A, Willis JR. Albuterol delivery by 4 different nebulizers placed in 4 different positions in a pediatric ventilator in vitro model. Respir Care 2013;58(7):1124-1133.

11. Hess D, Fisher D, Williams P, Pooler S, Kacmarek RM. Medication nebulizer performance: effects of diluent volume, nebulizer flow, and nebulizer brand. Chest 1996;110(2):498-505.

12. Ari A, Fink JB. Factors affecting bronchodilator delivery in mechanically ventilated adults. Nurs Crit Care 2010;15(4):192-203.

13. Gowda AA, Cuccia AD, Smaldone GC. Reliability of vibrating mesh technology. Respir Care 2017;62(1):65-69.

14. O'Doherty MJ, Thomas SH, Page CJ, Treacher DF, Nunan TO. Delivery of a nebulized aerosol to a lung model during mechanical ventilation. Am Rev Respir Dis 1992;146(2):383-388.

15. Aerogen. Aerogen Solo System Instruction Manual. Galway, Ireland: H. Aerogen, Ltd; 2017.

16. Cuccia AD, Ashraf S, McPeck M, Samuel J, Smaldone GC. Wet-side breath-enhanced jet nebulization: controlling drug delivery during mechanical ventilation. Respir Care 2020 [Epub ahead of print].

17. Ari A, Fink JB. Aerosol drug delivery during mechanical ventilation. Clin Pulm Med 2015;22(2):79-86.

18. Smaldone G, Solomita M. Predicting in vivo deposition in vitro. J Aerosol Med Pulm Drug Deliv 2009;22(1):9-10.

19. Palmer LB, Smaldone GC, Chen JJ, Baram D, Duan T, Monteforte M. Aerosolized antibiotics and ventilator-associated tracheobronchitis in the intensive care unit. Crit Care Med 2008;36(7):2008-2013.

20. Palmer LB, Smaldone GC, Simon S, O'Riordan T, Morra L. Tracheal aspirates in long-term mechanically ventilated patients: a human model of gram-negative infection and airway inflammation. Chest 1995;108(5):1326-1332

21. Palmer LB, Smaldone GC, Simon SR, O'Riordan TG, Cuccia A. Aerosolized antibiotics in mechanically ventilated patients: delivery and response. Crit Care Med 1998;26(1):31-39.

22. Ari A. Jet, ultrasonic, and mesh nebulizers: an evaluation of nebulizers for better clinical outcomes. Eurasian J Pulmonol 2014;16(1):1-7.

23. Ari A, Dornelas de Andrade A, Sheard M, AlHamad B, Fink J. Performance comparisons of jet and mesh nebulizers using different interfaces in simulated spontaneously breathing adults and children. J Aerosol Med Pulm Drug Deliv 2015;28(4):281-289.

24. Patel RB, Smaldone GC, Cuccia AD, Strachan P. In vitro delivery of aerosolized treprostinil via modern mechanical ventilation. J Aerosol Med Pulm Drug Deliv 2013;26(4):200-207.

This article is approved for Continuing Respiratory Care Education credit. For information and to obtain your CRCE

(free to AARC members) visit

www.rcjournal.com

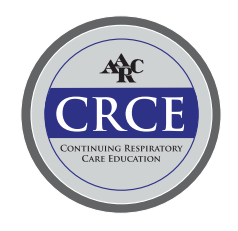

